\title{
Leakage Detection in Pipeline Based on Second Order Extended Kalman Filter Observer
}

\author{
S. Razvarz * R. Jafari ** C. Vargas-Jarillo* \\ A. Gegov $* * *$ M. Forooshani $* * *$ \\ * Departamento de Control Automatico, CINVESTAV-IPN (National Polytechnic Institute), \\ Mexico City07360,Mexico (E-mail: srazvarz@yahoo.com; cvargas@ctrl.cinvestav.mx) \\ ** School of design, University of Leeds, Leads, LS2 9JT, UK, \\ (Tel: +44 7493 205606; E-mail: r.jafari@leeds.ac.uk). \\ *** School of Computing, University of Portsmouth, Portsmouth, United Kingdom PO1 3BP, \\ (E-mail:alexander.gegov@port.ac.uk; massoud.forooshani@port.ac.uk)
}

\begin{abstract}
In this paper, a new technique is proposed in order to detect, locate, as well as approximate the fluid leaks in a straight pipeline (without branching) by taking into consideration the pressure and flow evaluations at the ends of pipeline on the basis of data fusion from two methods: a steady-state approximation and Second-order Extended Kalman Filter (SEKF). The SEKF is on the basis of the secondorder Taylor expansion of a nonlinear system unlike to the more popular First-order Extended Kalman Filter (FEKF). The suggested technique in this paper deals with just pressure head and flow rate evaluations at the ends of pipeline that has intrinsic sensor as well as process noise. A simulation example is given for demonstrating the validity of the proposed technique. It shows that the extended Kalman particle filter algorithm on the basis of the second-order Taylor expansion is effective and performs well in decreasing systematic deviations as well as running time.
\end{abstract}

(C) 2019, IFAC (International Federation of Automatic Control) Hosting by Elsevier Ltd. All rights reserved.

Keywords: Second-order Extended Kalman Filter; Leakage; Leakage detection; Pipeline

\section{INTRODUCTION}

Pipelines are extensively implemented in transferring great amounts of oil and gas products at large distances because of their safety, effectiveness as well as low cost. The protection of pipelines from theft and leakage is one of the primary aims of the oil and gas companies. Piping engineering is one of the fields that by monitoring the pipelines and using the methods such as artificial neural network (Yu, 2019) (Jafari, 2018, 2019b, 2019c), hydrostatic testing, infrared, and laser technology tries to found flaw in pipelines

Pipelines could suffer from various kinds of defects like corrosion, exhaustion cracks, dent, etc. Therefore, if these defects are not appropriately managed can cause pipeline failures having leak or rupture that may lead to extremely downtime and environment dangers (Verde, 2001, 2005) (Kowalczuk, 2000) (Garcia, 2009).

Automatic fault monitoring, as well as the detection in fluid distribution systems, are of great importance in today's world. The main aim of the automatic pipeline monitoring system is to diagnose leaks, obstructions or sensor faults as fast as feasible (Verde, 2017). Concerning leaks, these may lead to substantial economic damages, harm to the environs as well as health risks. There exist various techniques for the straight diagnosis of leaks relies on visual or palpable physical diagnosis of the fluid like hardware-based or computational pipeline monitoring techniques. Hardware-based techniques are highly based on the physical material installed through the pipeline. However, computational pipeline monitoring techniques are dependent on mathematical models of the pipeline. These techniques are complemented with measuring data of several physical variables related to the flow procedure e.g. pressure, flow rate, and temperature, between others. In the leak detection, apart from the detection of the leak, it is crucial to locate it as precise as feasible. Therefore, it is essential to implement algorithms, which can accurately diagnose the location of the leak since it is not all the time observable from outside the pipe.

The most current published researches are based on designing the observer by using the nonlinear filter or fault diagnosis techniques (Razvarz, 2019a, 2019b, 2019c, 2018) (jafari, 2019a). The functional form of the optimal nonlinear filter has long been recognized (Jazwinski, 1970), and usual techniques to executable algorithms are divided into two large groups. The first category contains algorithms, which estimate the nonlinear dynamical model and the second category contains algorithms, which estimate the latter distribution numerically, like the point mass filter as well as particle filter (Gustafsson, 2010). The most popular examples for the first category are the extended Kalman filter (EKF) (Athans, 1968), the unscented Kalman filter (UKF) (Julier, 1995) (Wan, 2000) and related sigma point techniques (Quine, 2006) like the recent quadrature Kalman filter (QKF) as well as cubature Kalman filter (CKF) (Arasaratnam, 2009). They all present the latter filtering distribution accompanied by a state approximation as 
well as an associated covariance matrix. The standard EKF is generally obtained from the first-order Taylor expansion of the state dynamics as well as measuring model. There exists another version of the EKF, which expands this approach to contain also an estimation of the second-order Taylor term (Jazwinski, 1970), (Arasaratnam,2009), (Anderson, 1979), that is known in past few decades. We will indicate the resulting algorithm SEKF to identify it from the standard algorithm.

For decreasing the error in the approximation of the system state and to increase the accuracy of EKF, different modified algorithms are proposed. In (Gao, 2014) an unscented Kalman filter (UKF) algorithm is suggested for nonlinear systems. The algorithm proposed in (Gao, 2014) contains the equal computation capacity like the EKF algorithm and also decreases the error in the linearizing nonlinear model. In (Qian, 2012) an algorithm named central difference Kalman filter (CDKF) is proposed and the recursive formula of the algorithm is deduced.

In this paper, a new technique is suggested for analyzing onedimensional modeling of transients in a pipeline, commonly used for recognition and location of leakage utilizing modelbased methods and SEKF observer. The SEKF algorithm is proposed for the nonlinear state-space model, which is based on second-order Taylor expansion of structural functions of the nonlinear system and applies a linearization technique to estimate the quadratic of second-order Taylor expansion of structural functions.

\section{PIPELINE MODELING}

In this work, we neglect the convective variation in velocity and the compressibility in the line of length $(\Gamma)$. The liquid density $(\rho)$, the flow rate $(\Phi)$, as well as pressure $(p)$ at the inlet and outlet of the pipeline are computable for assessment. The cross-sectional area (A) of the pipe is taken to be steady overall the pipe. The proposed pipeline is demonstrated in Figure 1.

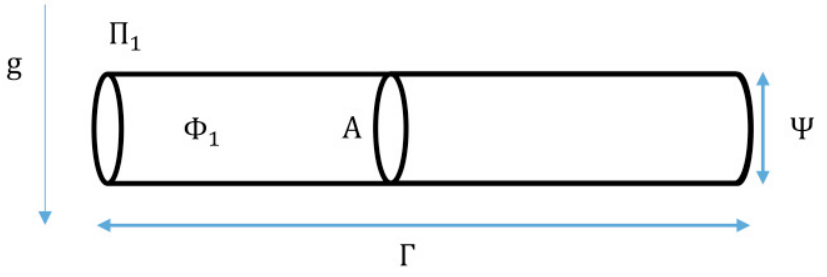

Fig. 1. The proposed pipeline system

The dynamics of fluid in the pipeline is described based on the mass and momentum, as well as the conservation (Brown, 2002). For obtaining the momentum equation by applying Newton's second law $(F=m a)$ to a control volume in the continuum and body force pipe $\left(s=\frac{f}{2 \Psi} v\right)$ the below formula is acquired (Brown, 2002),

$$
\frac{\partial v}{\partial t}+\frac{1}{\rho} \frac{\partial p}{\partial x}+\frac{f}{2 \Psi} v=0
$$

By replacing $v=\frac{\Phi}{A}$ and $p=\rho g \Pi$ in (1) the below formula is obtained,

$$
\frac{\partial \Phi}{A \partial t}+g \frac{\partial}{\partial x} \Pi+\frac{f \Phi}{2 \Psi \mathrm{A}^{2}}=0
$$

Therefore,

$$
\frac{\partial \Phi}{\partial t}+A g \frac{\partial}{\partial x} \Pi+\frac{f \Phi}{2 \Psi A}=0
$$

such that $\Pi$ is the pressure head, $\Phi$ is the flow rate, $x$ is the length coordinate, $t$ is the time coordinate, $g$ is the gravity, A is the section area, $\Psi$ is the diameter, and $f$ is the friction coefficient.

Generally, the friction coefficient is taken to be constant, although it is occasionally updated known to rely on the Reynolds number $(R e)$ and the roughness friction coefficient of the pipe $(e)$. The Swamee-Jain equation explains this friction coefficient value for a pipe with a circular section of diameter ( $\Psi)$ as below (P.K. Swamee, A.K. ain 1976),

$$
\mathrm{f}=\left(\frac{0.5}{\ln \left[0.27\left(\frac{e}{\Psi}\right)+5.74 \frac{1}{R e^{0.9}}\right]}\right)^{2}
$$

such that the Reynolds number is computed as follows:

$$
R e=4 \frac{\rho \Phi}{\pi \Psi \mu}=\frac{\rho v \Psi}{\mu}
$$

where $\rho$ is taken to be the fluid density also $\mu$ is taken to be the fluid viscosity. For $10^{-8}<\frac{e}{\Psi}<0.01$ and $5000<R e<10^{8}$, is valid.

$$
\frac{\partial p}{\partial t}+\rho a^{2} \frac{\partial v}{\partial x}=0
$$

In order to obtain the continuity equation, by implementing the law of conservation of mass and also utilizing the Reynolds transport theorem to a control volume and simplifying, the below formula is acquired,

$$
\begin{aligned}
& \frac{\partial p}{\partial t}+\rho a^{2} \frac{\partial v}{\partial x}=0 \\
& \frac{\partial p}{\partial t}+\rho a^{2} \frac{\partial v}{\partial x}=0
\end{aligned}
$$

By replacing the pressure head ( $\Pi$ ) and flow rate $(\Phi)$ in (8), the below formula is obtained,

$$
\frac{\partial \Pi}{\partial t}+\frac{a^{2}}{g A} \frac{\partial \Phi}{\partial x}=0
$$

such that $a$ is taken to be the velocity of the wave in an elastic conduit loaded with some compressible fluid. The velocity of the wave can be obtained from the elastic properties of the fluid as well as the pipe. The pressure head $(\Pi)$ and flow rate $(\Phi)$ are taken to be functions of position and time as $\Pi(x, t)$ and $\Phi(x, t)$, respectively, such that, $x \in[0, \Gamma]$, also, $\mathrm{L}$ is taken to be the length of the pipe. 
For a system having minor variations in the flow rate $\Phi$, the momentum formula from the nonlinear system is linearized as follows:

$$
\frac{\partial \Phi}{\partial t}+A g \frac{\partial}{\partial x} \Pi+\frac{f \Phi}{\Psi A}=0
$$

Using (9) and (10) we can design the pipeline model. However, acquiring the analytical solutions related to these equations is difficult. Different techniques are proposed for obtaining the numerical solutions for these equations such as characteristics and finite difference methods(Wylie, 1978). In this paper, we apply the finite difference technique.

For converting (9) and (10) into a system of ordinary differential equations, (9) and (10) are discretized in the spatial variable. The finite difference approach is considered as a discretization method that partitions the whole pipeline into $N$ number of sections (Beilina, 2016). The finite difference method with a constant step size $\Delta s$ is considered as the most common discretization technique in model-based techniques for real-time applications. In this paper, we use finite difference technique as it is easy to implement and is a proper technique for nonlinear observer design. The finite difference technique in this paper will be used as below,

$$
\begin{gathered}
\frac{\partial \Phi\left(s_{i-1}, t\right)}{\partial s} \approx \frac{\Delta \Phi\left(s_{i-1}, t\right)}{\Delta s} \approx \frac{\Phi_{i}-\Phi_{i-1}}{\Delta s} \\
\frac{\partial \Pi\left(s_{i-1}, t\right)}{\partial s} \approx \frac{\Delta \Pi\left(s_{i-1}, t\right)}{\Delta s} \approx \frac{\Pi_{i}-\Pi_{i-1}}{\Delta s}
\end{gathered}
$$

$\forall i=1, \cdots, n$, where $\mathrm{n}$ is the number of sections and $\Delta s=$ $s_{i+1}-s_{i}$ demonstrates the length of the $i$-section among two successive points of positions. The continuous interval $z \in$ $[0, \Gamma]$ is divided into a three-point discrete part $\left\{s_{k}\right\}:=$ $\left\{0, s_{\text {leak }}, \Gamma\right\}$, such that $z_{\text {leak }}$ demonstrates the indistinct leak position that shows in Figure 2. The flow rate of the leak is estimated with $\Phi_{\text {leak }}=C_{d} \mathrm{~A}_{\text {leak }} \sqrt{2 g} \sqrt{\Pi\left(s_{\text {leak }}, t\right)}$, where $C_{d}$ is the discharge coefficient, also $A_{\text {leak }}$ is the leak cross-section area. The leakage flow rate is obtained as $\Phi_{\text {leak }}=$ $\Lambda \sqrt{\Pi\left(s_{\text {leak }}, t\right)}$, where $\Lambda=C_{d} \mathrm{~A}_{\text {leak }} \sqrt{2 g}$. The dynamic model of the pipeline system is stated by an ordinary differential equation system as follows:

$$
\begin{aligned}
& \dot{\Phi}_{1}=\frac{g \mathrm{~A}}{s}\left(\Pi_{1}-\Pi_{2}\right)-\frac{f}{2 \mathrm{~A} \Psi} \Phi_{1} \\
& \dot{\Pi}_{\text {leak }}=\frac{c^{2}}{g \mathrm{~A} s}\left(\Phi_{1}-\Phi_{2}-\Lambda \sqrt{\Pi_{\text {leak }}}\right) \\
& \dot{\Phi}_{2}=\frac{g \mathrm{~A}}{\Gamma-s}\left(\Pi_{2}-\Pi_{3}\right)-\frac{f}{2 \mathrm{~A} \Psi} \Phi_{2}
\end{aligned}
$$

It has been assumed that the inlet pressure $h_{1}$ and the outlet pressure $h_{3}$ are known, which are externally defined by the pump power. The pressure $h_{2}$ at the leak point as well as the inlet and outlet flow rates $\left(\Phi_{1}\right.$ and $\left.\Phi_{2}\right)$ is taken to be the indistinct dynamic variable. According to the continuity equation in pipeline we have,

$$
\Phi_{1}=\Phi_{\text {leak }}+\Phi_{2}
$$

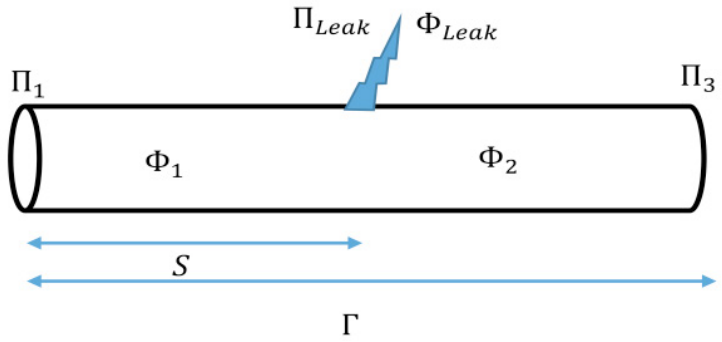

Fig. 2. Pipeline with leakage scheme

\section{OBSERVER DESIGN}

\subsection{Nonlinear State Space Model}

We will use filtering algorithms for the following nonlinear state space model,

$$
\begin{aligned}
& x_{i+1}=\varphi\left(x_{i}, u_{i}\right) \\
& y_{k}=\xi\left(x_{i}, e_{i}\right)
\end{aligned}
$$

where, $x_{i}$ is taken to be the $n_{x}$ dimensional state vector, $u_{i}$ and $e_{i}$ are the process and measurement noise vectors, respectively (dimensions $n_{u} \times 1$ and $n_{e} \times 1$, respectively), also, $y_{k}$ is the $n_{y}$-dimensional measured output vector. State transition $\varphi$ as well as measurement $\xi$ are considered as nonlinear functions. The process and measurement noise signals are supposed to be zero-mean Gaussian with definite covariance matrices $\breve{Q}_{i}$ and $\widetilde{R}_{l}$, respectively.

\subsection{System Approximation by Taylor Expansion}

The nonlinear state, as well as the measurement equation in (15), can be estimated using Taylor expansion around a specified state and a nominal (zero) noise value. Nevertheless, although derivative terms greater than order two are ignored in the expansion, the expressions are complicated. Therefore, we introduce new variables, which contain state and noise.

Defining $\mu_{i}=\left(x_{i}^{T}, u_{i}^{T}\right),(15)$ is rewritten when $x_{i+1}=\varphi\left(\mu_{i}\right)$. A Taylor expansion for each scalar element $x_{i+1}^{l}$ of the state vector $x_{i+1}$ around a known $\hat{\mu}_{i}$ is stated as below

$$
\begin{aligned}
& x_{i+1}^{l} \\
& \approx \varphi_{l}\left(\hat{\mu}_{i}\right)+\left(\nabla_{\mu} \varphi_{l}\left(\hat{\mu}_{i}\right)\right)^{T}\left(\mu_{i}-\hat{\mu}_{i}\right) \\
& +\frac{1}{2}\left(\mu_{i}-\hat{\mu}_{i}\right)^{T} \nabla_{\mu}^{2} \varphi_{l}\left(\hat{\mu}_{i}\right)\left(\mu_{i}-\hat{\mu}_{i}\right) \\
& l \in\left\{1, \ldots, n_{x}\right\}
\end{aligned}
$$

such that $\left(\nabla_{\mu} \varphi_{l}\left(\hat{\mu}_{i}\right)\right)^{T}$ is taken to be a row vector having the partial derivatives of $\varphi_{l}\left(\mu_{i}\right)$ regarding all elements of $\mu_{i}$ measured at $\hat{\mu}_{i}$. Consequently, $\nabla_{\mu}^{2} \varphi_{l}\left(\hat{\mu}_{i}\right)$ is a Hessian matrix having second order partial derivatives. Similarly, (16) is rewritten when $y_{i+1}=\xi\left(\varepsilon_{i}\right)$. A component of $y_{i}$ is defined as below, 


$$
\begin{aligned}
& y_{i}^{m} \\
& \approx \xi_{m}\left(\hat{\varepsilon}_{i}\right)+\left(\nabla_{\varepsilon} \xi_{m}\left(\hat{\varepsilon}_{i}\right)\right)^{T}\left(\varepsilon_{i}-\hat{\varepsilon}_{i}\right) \\
& +\frac{1}{2}\left(\varepsilon_{i}-\hat{\varepsilon}_{i}\right)^{T} \nabla_{\mu}^{2} \xi_{m}\left(\hat{\varepsilon}_{i}\right)\left(\varepsilon_{i}-\hat{\varepsilon}_{i}\right) \\
& \quad m \in\left\{1, \ldots, n_{y}\right\}
\end{aligned}
$$

The represented first as well as second order derivatives, for example, $\left(\nabla_{\mu} \varphi_{l}\left(\hat{\mu}_{i}\right)\right)^{T}$ or $\nabla_{\mu}^{2} \xi_{m}\left(\hat{\varepsilon}_{i}\right)$ are concatenated vectors and block matrices of derivatives with regard to $x$, and $u$ or $e$. Furthermore, mixed second order derivatives happen in the Hessians. Stacking, for example, all $n_{y}$ rows $\left(\nabla_{\varepsilon} \xi_{m}\left(\hat{\varepsilon}_{i}\right)\right)^{T}$ generates the Jacobian matrix $\left(\nabla_{x} \xi_{m}\left(\hat{\varepsilon}_{i}\right)\right)^{T}$ that will be utilized in the Kalman gain in the following subsection.

\subsection{Second Order Extended Kalman Filter Recursions}

Defining the initial state $x_{0}$ as well as its covariance matrix $P_{0}$, the filtering algorithm contains recursively named the time and measurement updates.

\subsubsection{Time Update}

A predicted approximation $\hat{x}_{i+1 \mid i}^{l}$ of $x_{i+1 \mid i}^{l}$ is obtained by implementing the expectation of as

$$
\begin{gathered}
\hat{x}_{i+1 \mid i}^{l}=\varphi_{l}\left(\hat{\mu}_{i \mid i}\right)+ \\
\frac{1}{2} \operatorname{tr}\left(\nabla_{x}^{2} \varphi_{l}\left(\hat{\mu}_{i \mid i}\right) \breve{P}_{i \mid i}\right)+\frac{1}{2} \operatorname{tr}\left(\nabla_{u}^{2} \varphi_{l}\left(\hat{\mu}_{i \mid i}\right) \check{Q}_{i}\right)
\end{gathered}
$$

It is supposed that $\hat{\mu}_{i \mid i}=\left(\hat{x}_{i \mid i}^{T}, 0^{T}\right)^{T}=E\left(\left(x_{i}^{T}, u_{i}^{T}\right)^{T} \mid y_{i}\right)$. Therefore, odd terms in $\mu_{i}$ disappear while executing the expectation. Moreover, the connection among $u_{i}$ and $x_{i}$ is not considered that can be stated by covariance matrix $\left(\mu_{i} \mid y_{i}\right)=$ Block diagonal matrix $\left(\breve{P}_{i \mid i}, \breve{Q}_{i}\right)$, hence no mixed second order derivatives happen in (19) (Tanizaki, 1996) (Hendeby, 2008). The predicted covariance matrix $P_{i+1 \mid i}=$ covariance matrix $\left(x_{i+1} \mid y_{i}\right)$ is defined by its elements in row $l$ and column $m$ as below,

$$
\begin{gathered}
\breve{P}_{i+1 \mid i}^{l m} \\
=\left(\nabla_{x} \varphi_{l}\left(\hat{\mu}_{i \mid i}\right)\right)^{T} \breve{P}_{i \mid i} \nabla_{x} \varphi_{m}\left(\hat{\mu}_{i \mid i}\right) \\
+\left(\nabla_{u} \varphi_{l}\left(\hat{\mu}_{i \mid i}\right)\right)^{T} \breve{Q}_{i} \nabla_{u} \varphi_{m}\left(\hat{\mu}_{i \mid i}\right) \\
+\frac{1}{2} \operatorname{tr}\left(\nabla_{x}^{2} \varphi_{l}\left(\hat{\mu}_{i \mid i}\right) \check{P}_{i \mid i} \nabla_{x}^{2} \varphi_{m}\left(\hat{\mu}_{i \mid i}\right) \check{P}_{i \mid i}\right) \\
+\frac{1}{2} \operatorname{tr}\left(\nabla_{u}^{2} \varphi_{l}\left(\hat{\mu}_{i \mid i}\right) \check{Q}_{i} \nabla_{u}^{2} \varphi_{m}\left(\hat{\mu}_{i \mid i}\right) \check{Q}_{i}\right)
\end{gathered}
$$

such that $l, m \in\left\{1, \ldots, n_{x}\right\}$. The covariance calculation is simple, however needs a Gaussian presumption on $x_{i}$.

\subsubsection{Measurement Update:}

carrying out the expectation of regarding a state prediction generates an output approximation as follows:

$$
\begin{gathered}
\hat{y}_{i \mid i-1}^{m} \\
=\xi_{m}\left(\hat{\varepsilon}_{i \mid i-1}\right)+\frac{1}{2} \operatorname{tr}\left(\nabla_{x}^{2} \xi_{m}\left(\hat{\varepsilon}_{i \mid i}\right) \check{P}_{i \mid i-1}\right) \\
+\frac{1}{2} \operatorname{tr}\left(\nabla_{e}^{2} \xi_{m}\left(\hat{\varepsilon}_{i \mid i}\right) \breve{R}_{i}\right)
\end{gathered}
$$

The output covariance matrix $\check{S}_{k}=$ covariance matrix $\left(y_{k} \mid y_{k-1}\right)$ is defined by its elements $l, m \in\left\{1, \ldots, n_{y}\right\}$ as follows:

$$
\begin{gathered}
\check{S}_{i}^{l m} \\
=\left(\nabla_{x} \xi_{l}\left(\hat{\varepsilon}_{i \mid i-1}\right)\right)^{T} \breve{P}_{i \mid i-1} \nabla_{x} \xi_{m}\left(\hat{\varepsilon}_{i \mid i-1}\right) \\
+\left(\nabla_{e} \xi_{l}\left(\hat{\varepsilon}_{i \mid i-1}\right)\right)^{T} \breve{R}_{i} \nabla_{e} \xi_{m}\left(\hat{\varepsilon}_{i \mid i-1}\right) \\
+\frac{1}{2} \operatorname{tr}\left(\nabla_{x}^{2} \xi_{l}\left(\hat{\varepsilon}_{i \mid i-1}\right) \breve{P}_{i \mid i-1} \nabla_{x}^{2} \xi_{m}\left(\hat{\varepsilon}_{i \mid i-1}\right) \breve{P}_{i \mid i-1}\right) \\
+\frac{1}{2} \operatorname{tr}\left(\nabla_{e}^{2} \varphi_{l}\left(\hat{\varepsilon}_{i \mid i-1}\right) \breve{R}_{i} \nabla_{e}^{2} \xi_{m}\left(\hat{\varepsilon}_{i \mid i-1}\right) \breve{R}_{i}\right)
\end{gathered}
$$

The uncorrelated noise has been supposed as $\operatorname{cov}\left(\varepsilon_{i} \mid y_{i-1}\right)=$ $\operatorname{blkdiag}\left(\breve{P}_{i \mid i-1}, \breve{R}_{i}\right)$. The Kalman gain is acquired under the supposition that $x_{i}$ and $y_{i}$ are jointly Gaussian distributed as stated by

$$
\left(\begin{array}{c}
x_{i} \\
y_{i}
\end{array}\right) \sim N\left(\left(\begin{array}{c}
\hat{x}_{i \mid i-1} \\
\hat{y}_{i \mid i-1}
\end{array}\right),\left(\begin{array}{cc}
\check{P}_{i \mid i-1} & \breve{M}_{i} \\
\breve{M}_{i}^{T} & \check{S}_{i}
\end{array}\right)\right)
$$

where the cross terms are defined as $\breve{M}_{i}=$ covariance matrix $\left(x_{i}, y_{i} \mid y_{i-1}\right)=\breve{P}_{i \mid i-1} \nabla_{x} \xi\left(\hat{\varepsilon}_{i \mid i-1}\right)$.

Applying optimal estimation theory (Anderson,1979), the posterior approximation is obtained as conditional expectation of $x_{i}$ as below,

$$
\hat{x}_{i \mid i}=\hat{x}_{i \mid i-1}+\breve{M}_{i} \check{S}_{i}^{-1}\left(y_{i}-\hat{y}_{i \mid i-1}\right)
$$

The iteration is finalized by updating the covariance matrix

$$
\breve{P}_{i \mid i}=\breve{P}_{i \mid i-1}+\breve{M}_{i} \breve{S}_{i}^{-1} \breve{M}_{i}^{T}
$$

Finally, $\breve{M}$ and $\breve{S}$ can be chosen as the covariance matrices of measure and process noises, respectively, or just as tuning parameters, with $\breve{P}=\breve{P}^{T}>0, \quad \breve{M}=\breve{M}^{T}>0$ and $\breve{S}=\breve{S}^{T}>$ 0

\section{SIMULATION RESULTS}

In this section, simulations results are reported for one case. The simulations are carried out by Matlab environment. The step time of the solver (ODE3) is set to $\Delta \mathrm{t}=0.001$. An illustration of the model is shown in Figure 1. The model has been realized on the pipeline with the following physical parameters:

The length of the pipeline is $\Gamma=120 \mathrm{~m}$, the diameter of the pipe is $\Psi=0.08 \mathrm{~m}$, the cross-section is $\mathrm{A}=5.03 \times 10^{-3} \mathrm{~m}^{2}$, density is $\rho=1000 \mathrm{~kg} / \mathrm{m}^{3}$, gravity is $g=9.81 \mathrm{~m} / \mathrm{s}^{2}$, the friction factor of the pipe is $f=0.06$, the friction factor of and the wave speed is $a=1250 \mathrm{~m} / \mathrm{s}$.

The covariance matrices of measure and process noises introduced by expressions respectively 
$\breve{M}$

$=$ Diagonal Matrix $\left[3.5 \times 10^{-5}, 5 \times 10^{-2}, 3.5\right.$

$\left.\times 10^{-5}, 1000,10^{-7}\right]$

$\check{S}=$ Diagonal Matrix $\left[3.5 \times 10^{-5}, 3.5 \times 10^{-5}\right]$

The initialization of the state is given in Table 1 .

Table 1. Initialization SEKF

\begin{tabular}{ccc}
\hline CEKF & value & units \\
\hline$\widehat{\Phi}_{1}$ & $7.75 \times 10^{-3}$ & $\left(\mathrm{~m}^{3} / \mathrm{s}\right)$ \\
$\widehat{\Pi}_{\text {leak }}$ & 8.32 & $(\mathrm{~m})$ \\
$\widehat{\Phi}_{2}$ & $7.75 \times 10^{-3}$ & $\left(\mathrm{~m}^{3} / \mathrm{s}\right)$ \\
$\hat{s}$ & 45.42 & $(\mathrm{~m})$ \\
$\widehat{\Lambda}$ & 0 & $\left(\mathrm{~m}^{\frac{5}{2}} / \mathrm{s}\right)$ \\
\hline
\end{tabular}

The pressure heads are shown in Figure. 3, and the flow rate are shown in Figure. 4 where it can be seen the effect of first leak at time $\mathrm{t}=30 \mathrm{~s}$,

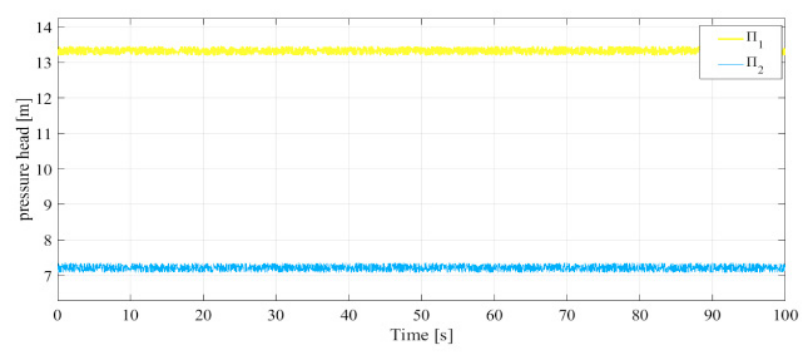

Fig.3. pressure heads at the pipeline

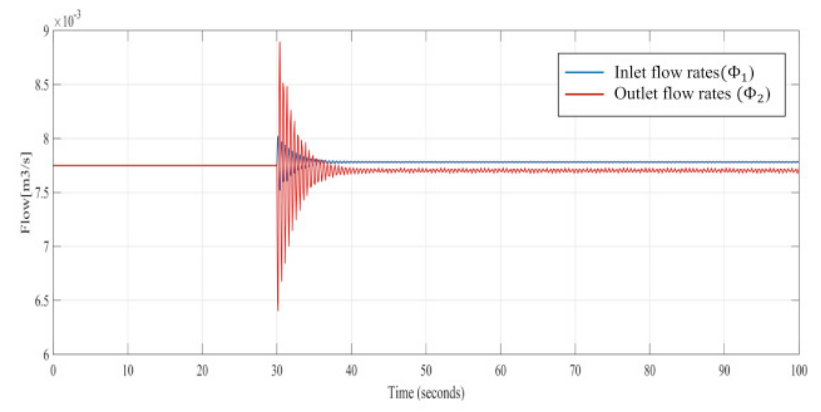

Fig. 4. The flow rate at the pipeline

The corresponding detection and isolation results are shown in Figures 5, 6 and 7. Figure 5 are shown, the measured pressure heads that correspond to leak points and shown, validate results obtained by observer

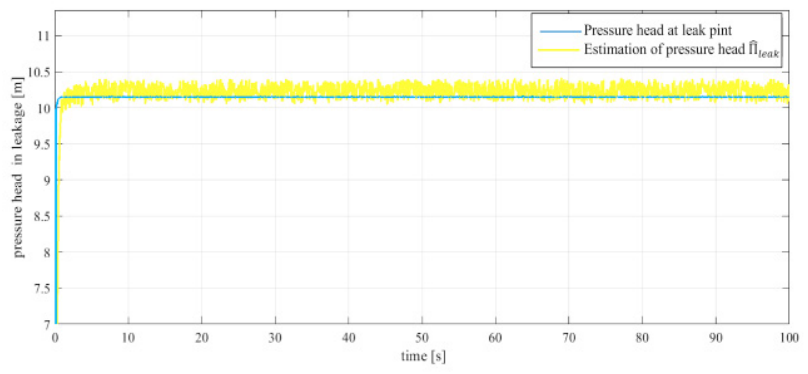

Fig. 5. Estimation of pressure head $\dot{\Pi}_{\text {leak }}$ and measurements of pressure head at leak point

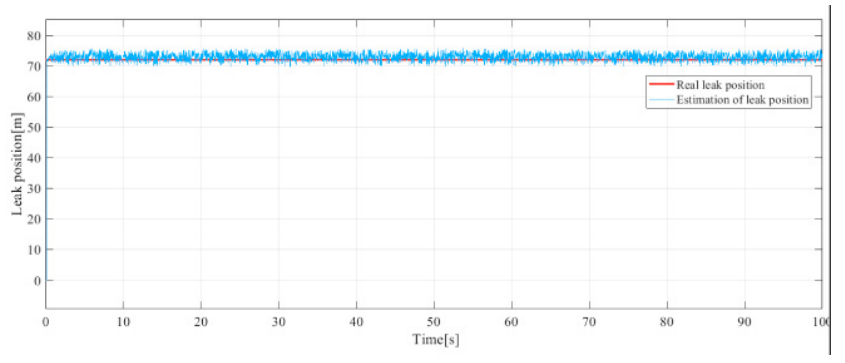

Fig. 6. Estimation and real leak position

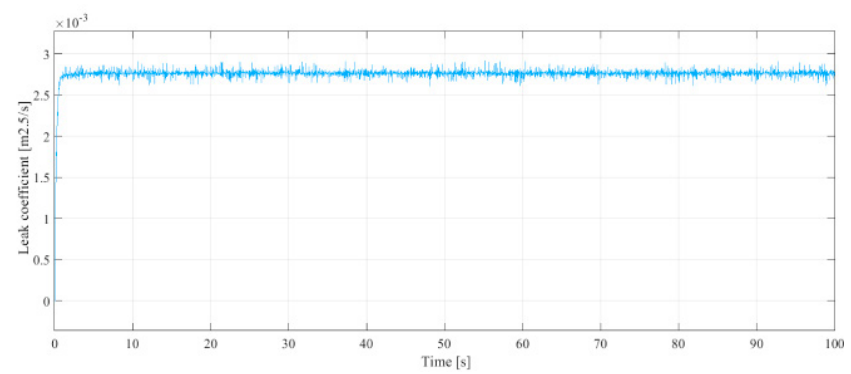

Fig. 7. Estimation of leak coefficients $\widehat{\Lambda}$

In Figure 6, is shown the estimated leak location. In Figure 7 the estimation leak coefficient is shown. In this case the leak identification is pretty successful.

\section{CONCLUSIONS}

In this paper, a technique based on SEKF is proposed to detect leakage in the pipeline. The SEKF algorithm is proposed for the nonlinear state-space model, which is based on secondorder Taylor expansion of structural functions of the nonlinear system and applies a linearization technique to estimate the quadratic of second-order Taylor expansion of structural functions. A simulation example is given for demonstrating the effectiveness of the proposed technique. In future research, the observability, as well as the controllability of the pipeline system will be investigated.

\section{REFERENCES}

Anderson. B. and Moore, J. B. (1979) Optimal Filtering, Prentice Hall.

Arasaratnam, I. and Haykin, S. (2009) Cubature Kalman filters, IEEE Transactions on Automatic Control, 54, 1254-1269.

Athans, M., Wishner, R. and Bertolini, A. (1968) Suboptimal state estimation for continuous-time nonlinear systems from discrete noisy measurements, IEEE Transactions on Automatic Control, 13, 504-514.

Beilina, L. (2016) Domain decomposition finite element/finite difference method for the conductivity reconstruction in a hyperbolic equation, Communications in Nonlinear Science and Numerical Simulation, 37, 222-237. 
Brown, G. (2002) The Darcy-Weisbach Equation, Oklahoma State University-Stillwate.

Gao. Y., Gao. S. and Wu, J. (2014) Fading-Memory SquareRoot Unscented Particle Filter Algorithm and Its Application in Integrated Navigation System, Journal of Chinese Inertial Technology, 22, 777-781.

Garcia, J., B. Leon. and O. Begovich. (2009) Validation of a semi-physical pipeline model for multi-leak diagnosis purposes, Int. Conf. on Modelling and Simulation, Alberta, Canada.

Golub, G. H. and Ortega J. M, (1992) Scientific Computing and Differential Equation: An introduction to numerical methods, Academic Press.

Gustafsson, F. (2010) Particle filter theory and practice with positioning applications, IEEE Aerospace and Electronic Systems Magazine, 25, 53-82.

Hendeby, G., (2008) Performance and implementaion aspects of nonlinear filtering, Linkoping Studies in Science and Technology. Dissertations, 1161.

Jafari, R., Razvarz, S., Gegov, A (2019) Neural Network Approach to Solving Fuzzy Nonlinear Equations using ZNumbers, IEEE Transactions on Fuzzy Systems. doi: 10.1109/TFUZZ.2019.2940919.

Jafari, R., Razvarz, S., Gegov, A. (2018) A New Computational Method for Solving Fully Fuzzy Nonlinear Systems. In: Computational Collective Intelligence. ICCCI 2018. Lecture Notes in Computer Science, Springer. 11055, pp 503-512. DOI: https://doi.org/10.1007/978-3319-98443-8-46 .

Jafari, R., Razvarz, S., Gegov, A. (2019) Solving Differential Equations with Z-Numbers by Utilizing Fuzzy Sumudu Transform. I IntelliSys 2018. Advances in Intelligent Systems and Computing, Springer, Cham, 869, pp. 11251138. https://doi.org/10.1007/978-3-030-01057-7.82.

Jafari, R., Razvarz. S., Vargas-Jarillo. C. and Yu, W. (2019) Control of Flow Rate in Pipeline Using PID Controller, 16th IEEE International Conference on Networking, Sensing and Control, (IEEE ICNSC 2019) May 9-11, 2019, Banff, Canada, 1, 293-298.

Jazwinski, A. H., (1970) Stochastic Processes and Filtering Theory, Academic Press.

Julier, S., Uhlmann, J. and Durrant-Whyte, H. (1995) A new approach for filtering nonlinear systems, Proceedings of the in American Control Conference, 3, 1628-1632.

Kowalczuk, K. and Gunawickrama, K. (2000) Leak detection and isolation for transmission pipelines via state estimation, IFAC Proceedings, 33, 921-926.

Qian, Z. and Qi, Y. (2012) A SLAM Algorithm Based on an Iterated Central Difference Particle Filter, Journal of Harbin Engineering University, 33, 355-360.

Quine, B. M. (2006) A derivative-free implementation of the extended Kalman filter, Automatica, 42, 1927-1934.

Razvarz, S., Vargas-Jarillo, C. and Jafari, R. (2019) Pipeline Monitoring Architecture Based on Observability and Controllability Analysis, 2019 IEEE International Conference on Mechatronics (ICM) Ilmenau, Germany, 1, 420-423.

Razvarz. S. and Jafari, R. (2018) Experimental Study of Al2O3 Nanofluids on the Thermal Efficiency of Curved Heat Pipe at Different Tilt Angle, Journal of Nanomaterials, Article ID 1591247, 1-7.

Razvarz. S., Jafari. R., and Vargas-jarillo. C. (2019) Modelling and Analysis of Flow Rate and Pressure Head in Pipelines 16th International Conference on Electrical Engineering, Computing Science and Automatic Control (CCE), Mexico City, Mexico, 1-6. doi: 10.1109/ICEEE.2019.8884587

Razvarz. S., Vargas-jarillo. C., Jafari. R. and Gegov. A. (2019) Flow Control of Fluid in Pipelines Using PID Controller, IEEE Access, 7, 25673-25680.

Swamee, P.K. and ain, A.K (1976) Explicit equations for pipeflow problems, Journal of the Hydraulics Division, 102, 657-664.

Tanizaki, H (1996) Nonlinear Filters: Estimation and Applications, 2nd ed. Springer, Springer.

Verde, C (2001). Multi - leak detection in fluid pipe lines. Control Engineering Practice, 9, 673-682

Verde, C (2005). Multi- leak isolation in a pipeline by unsteady state test. CDC-ECC Conf, Sevilla, Spain

Verde, C., Torres, L (2017) Modeling and monitoring of pipelines and networks: advanced tools for automatic monitoring and supervision of pipelines, Springer International Publishing,

Wan, E. and van der Merwe, R. (2000) The unscented Kalman filter for nonlinear estimation, in Adaptive Systems for Signal Processing, Communications, and Control Symposium, 153-158.

Wylie. E. B., and Streeter, V. L. (1978) Fluid Transient, New York City, New York, United States: McGraw-Hill Education.

Yu, W. and Jafari, R. (2019) Fuzzy Modeling and Control of Uncertain Nonlinear Systems (IEEE Press Series on Systems Science and Engineering) 1st Edition, 208 pages, Wiley-IEEE Press, ISBN: 978-1119491552. 